\title{
Adverse Drug Reaction Profile of SGLT2 Inhibitor-Associated Diabetic Ketosis/Ketoacidosis in Singapore and their Precipitating Factors
}

\author{
Michael Limenta ${ }^{1}$ (C) Christine S. C. Ho ${ }^{1} \cdot$ Jalene W. W. Poh ${ }^{1} \cdot$ Su-Yen Goh ${ }^{2} \cdot$ Dorothy S. L. Toh $^{1}$
}

Published online: 7 May 2019

(c) The Author(s) 2019

\begin{abstract}
Background and Objective As of December 2017, 20 diabetic ketosis (DK)/diabetic ketoacidosis (DKA) cases associated with sodium-glucose co-transporter 2 inhibitors (SGLT2i) had been reported to the Health Sciences Authority (HSA), Singapore. We aimed to provide a detailed analysis of the profile of these cases.

Methods As part of the emerging safety issue monitoring, the HSA followed up on SGLT2i-associated DK/DKA cases with the reporters to obtain the missing and/or supplementary information. Descriptive statistics were employed to summarise the data collected, while the Mann-Whitney test was employed to evaluate the differences between typical and euglycaemic DKA cases as well as between genders.

Results All cases led to hospitalisation but were non-fatal. Where reported, the majority (71-85\%) of DK/DKA cases occurred within 180 days of SGLT2i therapy initiation and involved female patients and/or patients with long-standing type 2 diabetes mellitus (T2DM). Apart from the difference in blood glucose levels, no differences in the profile between the typical and euglycaemic DKA cases were noted. Known precipitating factors were identified in all cases. Acute illnesses, particularly infections and abscesses, were the most commonly reported precipitating factors, followed by insulin dose reduction/cessation.

Conclusions Based on the profile of the reported cases, it is imperative to maintain clinical vigilance for DK/DKA, especially during the first 6 months of SGLT2i treatment and more so in female patients and/or patients with long-standing T2DM. Prompt evaluation and management of underlying precipitating factors is also important to assess and mitigate the risk of developing DK/DKA during treatment with SGLT2i.
\end{abstract}

\section{Key Points}

In the majority of cases, DK/DKA occurred within 180 days after initiation of SGLT2i therapy in female and/or long-standing T2DM patients with known precipitating factors.

The absolute number of SGLT2i-associated DK/DKA cases are expected to rise with the increasing use of SGLT2i.

The incidence of this serious but potentially preventable ADR could be reduced through appropriate clinical vigilance and patient education.

Michael Limenta

michael_limenta@hsa.gov.sg

1 Vigilance and Compliance Branch, Health Product Regulation Group, Health Science Authority, 11 Biopolis Way, \#11-01, Helios, Singapore 138667, Singapore

2 Department of Endocrinology, Singapore General Hospital, Outram Road, Singapore 169608, Singapore

\section{Introduction}

Sodium-glucose co-transporter 2 inhibitors (SGLT2is) are a relatively new class of oral glucose-lowering agents that first came on to the market in 2013. This class of agents works by increasing the renal excretion of glucose (i.e. glycosuria) through the inhibition of SGLT2-mediated renal glucose reabsorption, resulting in a reduction in plasma glucose concentration [1]. Despite their glucoselowering activity and cardiovascular benefits, SGLT2i administration has been associated with serious metabolic derangement referred to as diabetic ketosis (DK)/diabetic ketoacidosis (DKA). DKA is a potentially life-threatening condition that is typically characterised by hyperglycaemia, ketonaemia and anion-gap acidosis, while DK is diagnosed when there is ketonaemia and evidence for compensated metabolic acidosis (blood $\mathrm{pH}$ is within the normal range but with a decreased bicarbonate level). DK/DKA can also occur with lower than expected glucose levels (i.e. less than $13.9 \mathrm{mmol} / \mathrm{L}$-referred to as euglycaemic 
DK/DKA), possibly due to greater urinary loss of glucose or a decreased rate of hepatic glucose production [2, 3].

One of the postulated mechanisms for the development of DK/DKA during treatment with SGLT2i was inhibition of the activity of SGLT2 in the pancreatic alpha cells, which has been shown to trigger glucagon secretion [4]. Alteration in the glucagon-to-insulin ratio, together with pharmacologically induced glycosuria and precipitating factors, may synergistically promote development of DK/DKA. The known precipitating factors for DK/DKA include inadequate insulin treatment (including insulin treatment non-compliance), acute intercurrent illness (e.g. infection, myocardial infarction), low-carbohydrate diet and excessive alcohol intake [5].

The occurrence of increased risk of DK/DKA with SGLT2 $\mathrm{i}$ was detected in the post-marketing setting. A 2.2-fold increase in the risk of DKA was observed in a meta-analysis of three large post-marketing cardiovascular outcome trials of SGLT2i [6]. In addition, a recent study using a large claims database in the USA found that the incidence of DKA within 180 days following the initiation of SGLT2i was 2.2-fold higher than with dipeptidyl peptidase-4 inhibitors (DPP4is), the latter of which have no known association with DKA [7]. Despite their methodological limitations, published case reports and spontaneous case reports have made important contributions to the body of knowledge about the characteristics of this serious but potentially preventable adverse drug reaction (ADR) [8, 9]. However, the published literature on the characteristics of SGLT2i-associated DK/DKA in Asian patients, who are known to have a different diabetes profile or characteristics as compared to Caucasians [10], is relatively limited to date [11].

In Singapore, the first SGLT2i (canagliflozin) was approved in February 2014, followed by dapagliflozin in April 2014 and empagliflozin in December 2014. A local SGLT2i-associated DKA case was first reported to the Health Sciences Authority (HSA) of Singapore in October 2014 while a second DKA case with SGLT2is was reported in May 2015. The latter report coincided with the issuance of the US Food and Drug Administration (FDA)'s safety communication regarding this safety concern [12]. Subsequently, HSA initiated a benefit-risk assessment on SGLT2is and DKA, and communicated the assessment outcomes and recommendations to healthcare professionals (HCPs) in February 2016 [13]. HSA assessed that while the benefit-risk profile of SGLT2is remains favourable for their approved indications, the possibility of SGLT2i leading to an increased risk of DKA cannot be excluded, particularly in the presence of precipitating factors. Since then the number of DK/DKA cases associated with SGLT2is reported to the HSA has increased to 20 cases (as of December 2017).
In this article we provide a detailed analysis of the profile of SGLT2i-associated DK/DKA cases reported to the HSA, with a specific focus on the onset of the event and the precipitating factors. We envisage that these data will further add to the available body of knowledge surrounding this important serious adverse reaction.

\section{Methods}

The HSA, in its role as a national pharmacovigilance centre, receives reports of ADRs related to marketed health products from HCPs and product registrants. The ADR reports are submitted to the HSA via the Critical Medical Information Store (CMIS), email, online from HSA's website, fax or mail. The CMIS is a data repository for ADRs, drug allergies and medical alerts that allows healthcare professionals to enter ADRs into patients' electronic medical records, and the information is then transmitted electronically to the HSA.

As part of monitoring of the emerging safety issue, for reported SGLT2i-associated DK/DKA cases, the HSA followed up with the reporting $\mathrm{HCP}$ or product registrant to obtain additional information, if they were not described in the initial reports, to have a complete case report. These include, but are not limited to, information such as the patient's age, body mass index (BMI), gender, diabetes type (e.g. type 1 or type 2) and duration, time to onset of DK/ DKA, suspected and concomitant drugs, outcome of the DK/ DKA event, relevant laboratory results (i.e. blood glucose, $\mathrm{HbA1c}$, bicarbonate, blood/urine $\mathrm{pH}$ and ketone levels) and concurrent medical conditions that may precipitate the development of DK/DKA.

Descriptive statistics were employed to summarise the data collected. The Mann-Whitney test was performed to evaluate if the differences between the characteristics of DKA and euglycaemic DKA cases are statistically significant as well as evaluate gender-related differences. Continuous variable data were expressed as median and range (minimum and maximum values). A $P$ value $<0.05$ was considered statistically significant. Local exposure to SGLT2 is was estimated from national sales data, which were expressed as the number of defined daily doses (DDDs). DDD is defined as the assumed average maintenance adult dose per day [14]. The reporting rate of SGLT2i-associated DK/DKA was calculated as a ratio of the number of DK/ DKA cases to the number of DDDs in the same period and expressed as number of cases per million DDDs (annual reporting rate) or number of cases per 1000 DDD-years (cumulative reporting rate). 


\section{Results}

As of December 2017, a total of 20 reports of SGLT2i-associated DK/DKA had been received by the HSA, of which the majority $(80 \%)$ were reported by healthcare professionals while $20 \%$ were reported by product registrants. All the reported cases were serious and resulted in hospitalisation but none were fatal. DKA was described in 17 reports while DK was described in the remaining three reports. As the number of DK reports was too small to perform meaningful analyses, the data from DKA and DK reports were combined and analysed simultaneously, unless otherwise stated.

The clinical characteristics of these cases are shown in Table 1 . There were no statistically significant gender differences in the clinical characteristics, except for BMI (data not shown). Other than the blood glucose level, none of the differences in clinical characteristics between the euglycaemic and typical DKA cases were statistically significant (data not shown).

\subsection{Demographics}

Of the 20 reports, $75 \%$ involved female patients and $25 \%$ involved male patients, with age ranging from 22 to 77 years (median 53.5 years). Ethnicity was reported in 19 reports, of which $89 \%$ were ethnic Chinese patients. BMI data were available for 15 reports (median $27.6 \mathrm{~kg} / \mathrm{m}^{2}$; range $15.7-34.7 \mathrm{~kg} / \mathrm{m}^{2}$ ), of which one was severely underweight $\left(\mathrm{BMI} 15.7 \mathrm{~kg} / \mathrm{m}^{2}\right)$. The majority of the reports $(90 \%)$ involved patients with type 2 diabetes mellitus (T2DM). There were two reports that involved patients with latent autoimmune adult diabetes (LADA). The duration of T2DM was available for 14 reports, of which ten had T2DM for at least 8 years.

\subsection{SGLT2i Therapies}

Canagliflozin and empagliflozin were the suspected agents in eight and seven reports, respectively. Dapagliflozin was the suspected agent in six reports, including one report that involved both dapagliflozin and empagliflozin. The time from drug initiation to DK/DKA onset (denoted time-toonset; TTO) was available for 19 reports (median 50 days, range 4-789 days), of which 85\% developed DK/DKA within 180 days after SGLT2 inhibitor therapy initiation, as shown in Fig. 1. Metformin was the most commonly reported concomitant antidiabetic drug, reported in $75 \%$ of the cases, followed by DPP-4is (45\%), sulfonylureas (25\%) and insulin (20\%).

\subsection{Laboratory Results}

Glycated hemoglobin (HbA1c) levels were available for 15 reports. Of these 15 reports, seven had $\mathrm{HbA} 1 \mathrm{c}$ levels reported between $8.0 \%$ and $10.0 \%$ while the remaining
Table 1 Clinical characteristics of diabetic ketosis (DK)/diabetic ketoacidosis (DKA) cases reported to the Health Sciences Authority (HSA)

\begin{tabular}{ll}
\hline Characteristics & Value \\
\hline Reporter & \\
Healthcare professionals & $16(80)$ \\
Product registrants & $4(20)$ \\
ADR description & \\
DK & $3(15)$ \\
DKA & $9(45)$ \\
Euglycaemic DKA & $8(40)$ \\
Demographics & \\
Males/females & $5(25) / 15(75)$ \\
T2DM/LADA & $18(90) / 2(10)$ \\
Diabetes duration (years) & $14($ newly diagnosed-30; 14) \\
Age (years) & $53.5(22-77 ; 20)$ \\
BMI (kg/m $\left.{ }^{2}\right)$ & $27.6(15.7-34.7 ; 15)$ \\
Suspected SGLT2i & \\
Canagliflozin & $7(33.3)$ \\
Dapagliflozin & $6^{\mathrm{a}}(28.6)$ \\
Empagliflozin & $8^{\mathrm{a}}(38.1)$ \\
TTO (days) & $50(4-789 ; 19)$ \\
Concomitant anti-diabetic drugs & \\
Metformin & $15(75)$ \\
DPP4i & $9(45)$ \\
Sulfonylurea & $5(25)$ \\
Insulin & $4(20)$ \\
Laboratory tests & \\
Blood glucose (mmol/L) & $14.3(8.1-25.5 ; 20)$ \\
BbA1c (\%) & $10.2(8-16 ; 15)$ \\
Bicarbonate (mmol/L) & $7.27\left(5^{\mathrm{b}}-7.42 ; 16\right)$ \\
Blood ketone (mmol/L) & $10(2.4-21.3 ; 20)$ \\
\hline & $5(1.6-6.8 ; 16)$ \\
\hline & \\
Positive urinary ketone & \\
\hline
\end{tabular}

Values are expressed as $n(\%)$ or median (range; $n$ )

$B M I$ body mass index, HbAlc glycated hemoglobin, TTO time to onset, T2DM type 2 diabetes mellitus, SGLT2i sodium-glucose cotransporter 2 inhibitor, DPP4i dipeptidyl peptidase-4 inhibitor

${ }^{a}$ One case reported both dapagliflozin and empagliflozin

${ }^{\mathrm{b}}$ One case reported a urine $\mathrm{pH}$ of 5

eight reported $\mathrm{HbA} 1 \mathrm{c}$ levels $>10.0 \%$. Serum bicarbonate levels were available for all reports, where ten had bicarbonate levels $<10 \mathrm{mmol} / \mathrm{L}$ while seven had bicarbonate levels between $10 \mathrm{mmol} / \mathrm{L}$ and $<18 \mathrm{mmol} / \mathrm{L}$. The remaining three reports, which were DK cases, had bicarbonate levels of $18 \mathrm{mmol} / \mathrm{L}$ and above. Blood and urine $\mathrm{pH}$ levels were available for 15 reports and one report, respectively. Five of the reports had blood $\mathrm{pH}$ levels $<7.20$ while another five reports had blood $\mathrm{pH}$ levels reported between 7.20 and 7.35. The remaining five reports had blood $\mathrm{pH}$ levels $>7.35$. Blood ketone levels were available for 


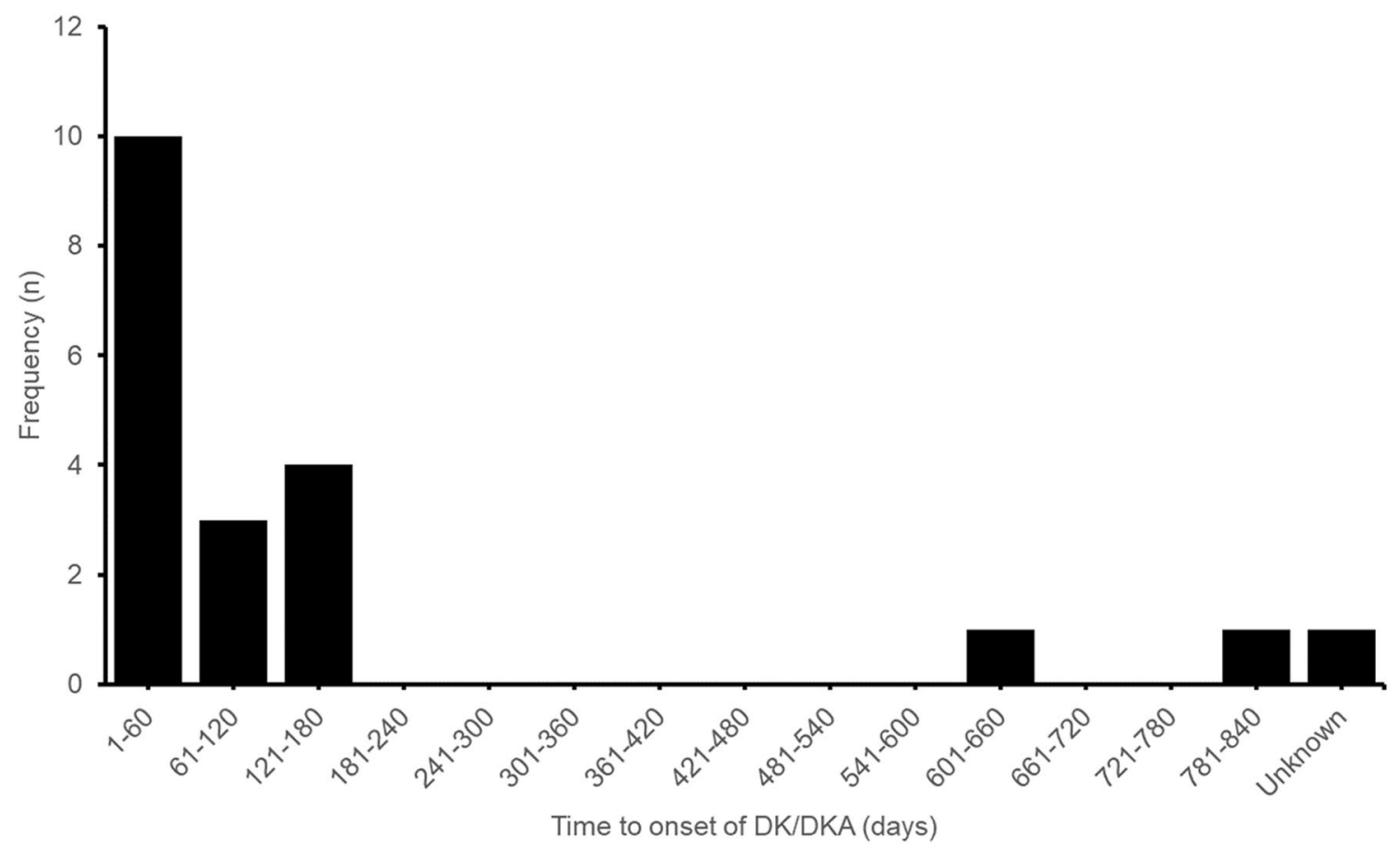

Fig. 1 Time from sodium-glucose co-transporter 2 inhibitor (SGLT2i) treatment initiation to DK/DKA onset. $D K$ diabetic ketosis, $D K A$ diabetic ketoacidosis

16 reports, ranging from $1.6 \mathrm{mmol} / \mathrm{L}$ to $6.8 \mathrm{mmol} / \mathrm{L}$ (median $5 \mathrm{mmol} / \mathrm{L}$ ), while a positive urine ketone test was described in the remaining four reports. Blood glucose levels were available for all reports (median $14.3 \mathrm{mmol} / \mathrm{L}$; range 8.1-25.5 mmol/L), half of which had glucose levels $<13.9 \mathrm{mmol} / \mathrm{L}$.

\subsection{Precipitating Factors}

Known DKA precipitating factors were described in all reports. Acute illnesses were the most commonly reported DKA precipitating factors (reported in $70 \%$ of the cases), followed by insulin dose reduction/cessation (15\%), as shown in Fig. 2. The reported acute illnesses included urinary tract infection (UTI), upper respiratory tract infection, abscesses at various sites, and fracture. UTI was described in four reports involving three female patients and one male patient. The other DKA precipitating factors included alcoholism, low medication adherence, low BMI, low carbohydrate diet and Ramadhan (Islamic holy month) fasting.

\subsection{Estimated Local Exposure to SGLT2is and Reporting Rate of SGLT2i-Associated DK/ DKA}

Local exposure to SGLT2 is saw an increasing trend from 2014 to 2017. The number of DDDs increased from 0.6 million in 2014 to 5 million in 2017. Annual reporting rates of SGLT2i-associated DK/DKA initially increased from 1.8 cases per million DDDs in 2014 to 2.8 cases per million DDDs in 2016. However, the reporting rate in 2017 decreased to 1.6 cases per million DDDs, which was even slightly lower than that in 2014 (Fig. 3). The cumulative reporting rate of SGLT2i-associated DK/DKA was 0.76 cases per 1000 DDD-years. Individually, the cumulative reporting rates of DK/DKA associated with the use of canagliflozin, dapagliflozin and empagliflozin were 1.12, 0.47 and 0.95 cases per 1000 DDD-years, respectively.

\section{Discussion}

Published case reports as well as spontaneous case reports have collectively contributed to a better understanding of the safety profile of SGLT2i-associated DKA. Recent studies by Fadini and colleagues provided a detailed analysis of SGLT2i-associated DKA reports in the US FDA Adverse Event Reporting System (FAERS) as well as in 105 published case reports [8,9]. We discuss the significance of our findings in relation to their findings.

Highly variable time-to-onset (TTO; time from drug initiation to onset of DKA) of DKA was observed in FAERS reports (range 1 day to $>8$ years) as well as published case reports (0.3-420 days) [8,9]. Based on Bonora et al.'s 


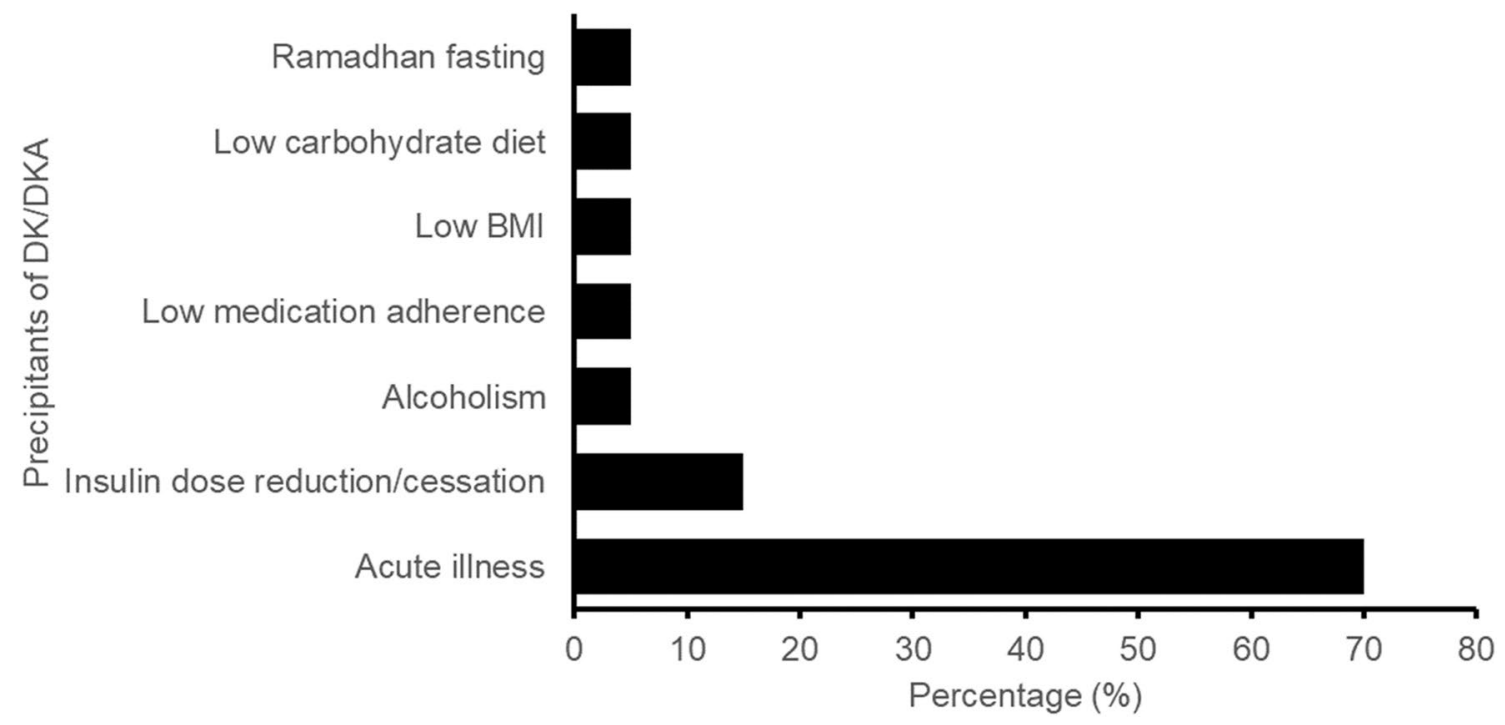

Fig. 2 Frequency of the identified precipitants of DK/DKA. $D K$ diabetic ketosis, $D K A$ diabetic ketoacidosis, $B M I$ body mass index

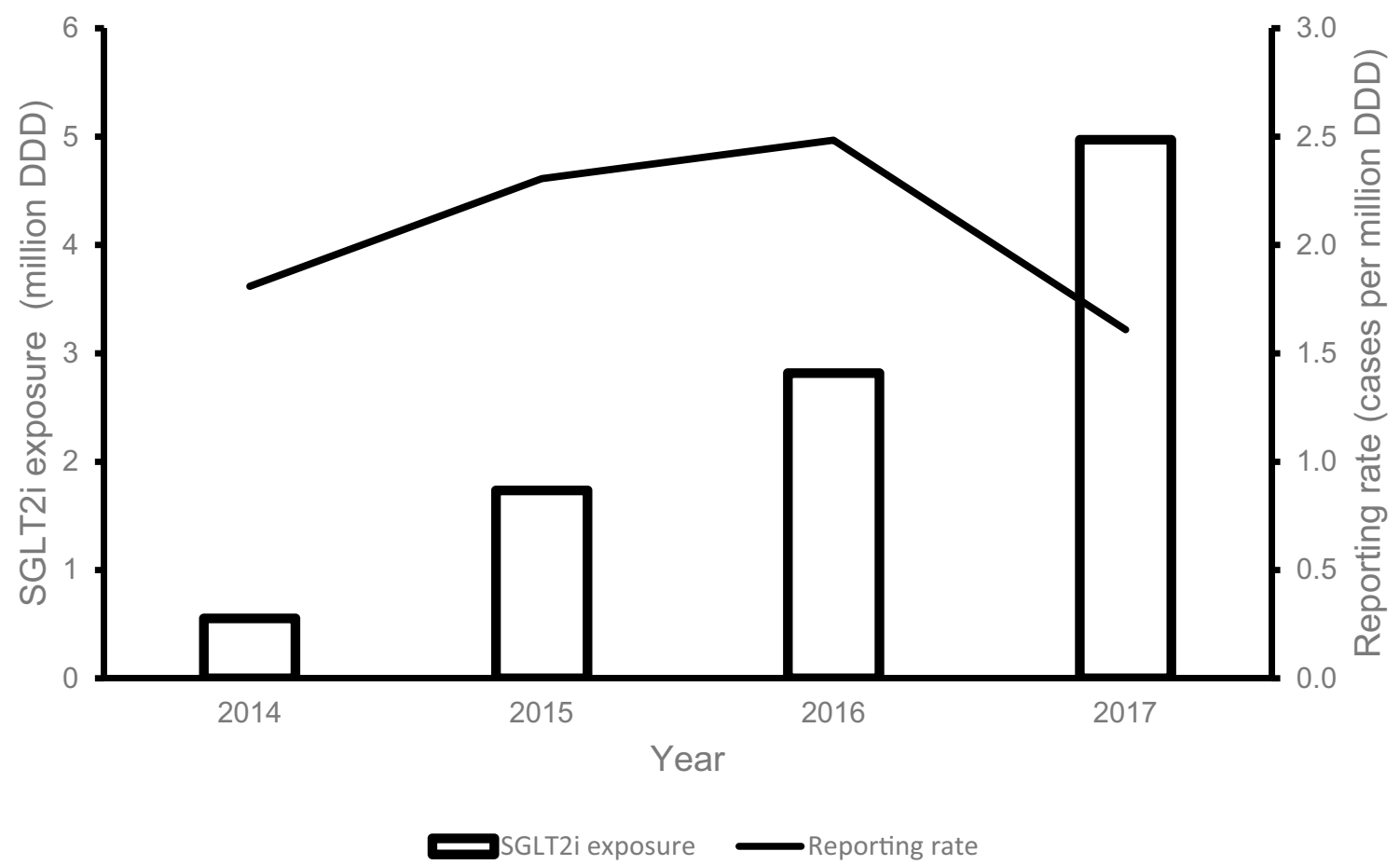

Fig. 3 Sodium-glucose co-transporter 2 inhibitor (SGLT2i) exposure and reporting rate of SGLT2i-associated DK/DKA. DK diabetic ketosis, $D K A$ diabetic ketoacidosis, $D D D$ defined daily dose

published supplementary information, we noted that the majority $(84.8 \%)$ of the published case reports with information on TTO of DKA $(n=46)$ occurred within 180 days of initiation of SGLT2 $i$ therapy. This is similar to our findings, which may suggest that patients may be at higher risk of developing DKA during the first 180 days of SGLT2i therapy (Fig. 1). Few studies have provided evidence that the risk of developing DKA decreases with a longer duration of SGLT2i exposure. In one Korean nationwide population-based cohort study by Kim et al., a decrease in the 
cumulative incidence of DKA during the first 3 years after SGLT2 $i$ therapy initiation compared with the incidence during the first 180 days after initiation ( 0.614 vs. 1.073 per 1000 person-years) was observed [15]. However, their study results also indicated that SGLT2i use does not increase the risk of DKA compared with DPP4i use. In contrast, Fralick et al. examined a large claims database of commercially insured patients in the USA and found that patients treated with SGLT2is have about twice the risk for developing DKA within 180 days of therapy initiation compared to those treated with DPP4is [7]. The 180-day cumulative incidence of DKA reported by Fralick et al. was also 4.6-fold higher than that reported by Kim et al. (4.9 vs. 1.073 per 1000 person-years). Additional well-designed studies are warranted to further characterise the risk of DKA with SGLT2is stratified by drug exposure duration.

Consistent with the findings of Fadini et al. and Bonora et al. (female-to-male ratio of up to 1.57), female gender predominance was also observed in our cases, albeit with a higher female-to-male ratio of 3 . The reasons for this phenomenon are unclear. It is known that genital infection and UTI are more prevalent in women during treatment with SGLT2is, and can trigger DKA. Although there were no cases reported to us that described genital infections, there were four reports that described UTIs and the majority of them involved females. There is currently no evidence to suggest gender differences in the prescribing of SGLT2is [16]. It is noteworthy that there was a difference in the TTO of DKA, albeit statistically insignificant $(P=0.06)$, between male patients (median 13 days; range 8-43 days) and female patients (median 120 days; range 4-789 days), suggesting that DKA may develop earlier in male patients than in female patients following initiation of SGLT2i therapy. Gender differences in the susceptibility to DKA during SGLT2i therapy should be further explored.

Acute illnesses, mainly infections and abscesses, were the most common precipitant of DKA in our cases (Fig. 2). By contrast, relative/absolute insulin deficit was the most common DKA precipitant present in the published case reports reviewed by Bonora et al. [9]. This may partly be due to a higher prevalence of concomitant insulin use and T1DM in the published case reports compared to our cases (40\% vs. $20 \%$ and $27 \%$ vs. none, respectively). It has been shown that diabetic patients are at increased risk for recurrent common infections, such as lower respiratory tract infections, UTIs, and skin and mucous membrane infections, and are vulnerable to recurrent severe infections, potentially due to altered innate and adaptive immunity [17-19]. Metabolic responses to infection, such as elevated glucagon and catecholamine concentrations and insulin resistance, may in turn increase patients' susceptibility to DKA [20]. In addition, where reported, 71\% of our cases involved patients with long-standing DM (DM duration of 8 years and above). Due to the progressive nature of the disease, long-standing T2DM patients are at risk of developing insulin deficiency over time, putting them at risk of developing DKA during SGLT2i treatment [21]. Prudent SGLT2i prescribing in these patients is essential.

Euglycaemic DKA contributed to nearly half of the cases (40\%) reported to us. Apart from the blood glucose levels, no statistically significant differences in clinical characteristics between the typical and euglycaemic DKA cases were observed. Compared to typical DKA cases, euglycaemic DKA cases tended to have shorter TTOs of DKA, albeit being statistically insignificant ( $P=0.13$; data not shown). Also, we observed that euglycaemic DKA cases tend to involve patients with a shorter duration of DM. However, statistical analysis was not performed as the DM duration data were only available for four euglycaemic DKA cases, three of which were described as 'newly diagnosed' DM. To our knowledge, no studies to date have examined the extent to which the profiles of SGLT2i-associated euglycaemic DKA cases differ from those of SGLT2i-associated typical DKA. More cases are required to validate these findings.

As of December 2017, the cumulative reporting rate of SGLT2i-associated DK/DKA was 0.72 cases per 1000 DDDyears, which translates to 0.72 cases per 1000 person-years. The cumulative reporting rates of DK/DKA associated with dapagliflozin, empagliflozin and canagliflozin were 0.47 cases per 1000 DDD-years, 0.95 cases per 1000 DDD-years and 1.12 cases per 1000 DDD-years, respectively. These rates fall within the range previously reported for the DKA incidence rate in SGLT2i users (0.6-1.69 cases per 1000 personyears) as well as in T2DM (0.32-2.0 cases per 1000 personyears) [15, 22-25]. Despite considerable increases in the use of SGLT2is from 2014 to 2017, a parallel increase in annual reporting rates of SGLT2i-associated DK/DKA was not observed. The annual reporting rate of SGLT2i-associated DK/DKA initially increased during the 2014-2016 period but declined in 2017 (Fig. 3). It remains to be seen whether the annual reporting rates will remain low. Also, it should be noted that, due to the voluntary nature of ADR reporting by HCPs and the use of sales data to estimate local exposure to SGLT2is, these reporting rates may be an under- or overestimation of the true rates. Nonetheless, profiling of the reported cases helps to provide a collective understanding of the predisposing and precipitating factors for DK/DKA in SGLT2i-treated patients, which is the cornerstone in determining the risk minimisation measures that can be put in place to reduce its severity and/or probability of occurrence.

\section{Conclusion}

The absolute number of SGLT2i-associated DK/DKA cases are expected to rise with the increasing use of SGLT2is. The incidence of this serious but potentially preventable ADR 
could be reduced through appropriate clinical vigilance and patient education. It is imperative to maintain clinical vigilance for DKA, especially during the first 6 months of treatment with SGLT2is, and more so in female patients and/ or patients with long-standing type 2 diabetes. Prompt evaluation and management of underlying precipitating factors is also important to assess and mitigate the risk of developing DK/DKA during treatment with SGLT2is. Patients on SGLT2is should be advised about possible DK/DKA episodes and their precipitating factors, and to seek medical attention promptly upon experiencing symptoms of metabolic acidosis.

Acknowledgements We would like to express our appreciation to healthcare professionals (HCPs) and product registrants for reporting the SGLT2i-associated DK/DKA cases, and for providing additional information. We thank the Adverse Event Management Unit for assisting with obtaining additional information from the reporting HCPs and product registrants. We also thank IQVIA for providing the national sales data for SGLT2i-containing products.

Data Availability The datasets analysed in this study are available in the HSA Adverse Event Online Database, which is accessible by local industry partners and HCPs.

\section{Compliance with Ethical Standards}

Funding No sources of funding were used to conduct this study. Singapore General Hospital paid the open access fee.

Conflict of interest Michael Limenta, Christine S. C. Ho, Jalene W. W. Poh, Su-Yen Goh and Dorothy S. L. Toh have no conflicts of interest that are directly relevant to the content of this article. The views expressed in this article are the personal views of the authors and may not be understood or quoted as being made on behalf of or reflecting the position of the Health Sciences Authority or any of its committees or working parties.

Ethics approval and informed consent Ethics approval and informed consent are not required for national pharmacovigilance activities that do not involve hypothesis testing and systematic investigation.

Open Access This article is distributed under the terms of the Creative Commons Attribution-NonCommercial 4.0 International License (http://creativecommons.org/licenses/by-nc/4.0/), which permits any noncommercial use, distribution, and reproduction in any medium, provided you give appropriate credit to the original author(s) and the source, provide a link to the Creative Commons license, and indicate if changes were made.

\section{References}

1. Chao EC, Henry RR. SGLT2 inhibition-a novel strategy for diabetes treatment. Nat Rev Drug Discov. 2010;9(7):551-9. https:// doi.org/10.1038/nrd3180.

2. De P, Child DF. Euglycaemic diabetic ketoacidosis-is it on the rise? Pract Diabetes Int. 2001;18:239-40.
3. Rjf E. Euglycemic diabetic ketoacidosis: a predictable, detectable, and preventable safety concern with SGLT2 inhibitors. Diabetes Care. 2015;38(9):1638-42.

4. Bonner C, Kerr-Conte J, Gmyr V, Queniat G, Moerman E, Thevenet J, et al. Inhibition of the glucose transporter SGLT2 with dapagliflozin in pancreatic alpha cells triggers glucagon secretion. Nat Med. 2015;21(5):512-7. https://doi.org/10.1038/nm.3828.

5. Kitabchi AE, Hirsch IB, Emmet M. Diabetic ketoacidosis and hyperosmolar hyperglycemic state in adults: clinical features, evaluation, and diagnosis. In: Post TW, editor. UpToDate. Waltham: UpToDate; 2018.

6. Zelniker TA, Wiviott SD, Raz I, Im K, Goodrich EL, Bonaca MP, et al. SGLT2 inhibitors for primary and secondary prevention of cardiovascular and renal outcomes in type 2 diabetes: a systematic review and meta-analysis of cardiovascular outcome trials. Lancet. 2019;393:31-9.

7. Fralick M, Schneeweiss S, Patorno E. Risk of diabetic ketoacidosis after initiation of an SGLT2 inhibitor. N Engl J Med. 2017;376(23):2300-2. https://doi.org/10.1056/NEJMc1701990.

8. Fadini GP, Bonora BM, Avogaro A. SGLT2 inhibitors and diabetic ketoacidosis: data from the FDA Adverse Event Reporting System. Diabetologia. 2017;60(8):1385-9. https://doi.org/10.1007/ s00125-017-4301-8.

9. Bonora BM, Avogaro A, Fadini GP. Sodium-glucose co-transporter-2 inhibitors and diabetic ketoacidosis: an updated review of the literature. Diabetes Obes Metab. 2018;20(1):25-33. https ://doi.org/10.1111/dom.13012.

10. Rhee EJ. Diabetes in Asians. Endocrinol Metab (Seoul). 2015;30(3):263-9. https://doi.org/10.3803/EnM.2015.30.3.263.

11. Ogawa W, Sakaguchi K. Euglycemic diabetic ketoacidosis induced by SGLT2 inhibitors: possible mechanism and contributing factors. J Diabetes Investig. 2016;7(2):135-8. https://doi. org/10.1111/jdi.12401.

12. FDA warns that SGLT2 inhibitors for diabetes may result in a serious condition of too much acid in the blood; 2015. https://www. fda.gov/downloads/drugs/drugsafety/ucm446954.pdf. Accessed 2 Jan 2019.

13. Risk of diabetic ketoacidosis associated with sodium-glucose cotransporter-2 inhibitors; 2016. https://www.hsa.gov.sg/conte $\mathrm{nt} / \mathrm{hsa} / \mathrm{en} /$ Health_Products_Regulation/Safety_Information_and_ Product_Recalls/Product_Safety_Alerts/2016/sodium-glucosecotransporter2sglt2inhibitorsandriskofseriousdiab.html. Accessed 2 Jan 2019.

14. ATC/DDD index. WHO Collaborating Centre for Drug Statistics Methodology; 2018. http://www.whocc.no/atcddd/. Accessed 2 Jan 2019.

15. Kim YG, Jeon JY, Han SJ, Kim DJ, Lee KW, Kim HJ. Sodiumglucose co-transporter-2 inhibitors and the risk of ketoacidosis in patients with type 2 diabetes mellitus: a nationwide population-based cohort study. Diabetes Obes Metab. 2018. https://doi. org/10.1111/dom.13297.

16. Kosiborod M, Lam CSP, Kohsaka S, Kim DJ, Karasik A, Shaw J, et al. Cardiovascular events associated with SGLT-2 inhibitors versus other glucose-lowering drugs: the CVD-REAL 2 study. $\mathrm{J}$ Am Coll Cardiol. 2018;71(23):2628-39.

17. Muller LM, Gorter KJ, Hak E, Goudzwaard WL, Schellevis FG, Hoepelman AI, et al. Increased risk of common infections in patients with type 1 and type 2 diabetes mellitus. Clin Infect Dis. 2005;41(3):281-8. https://doi.org/10.1086/431587.

18. Hamilton EJ, Martin N, Makepeace A, Sillars BA, Davis WA, Davis TM. Incidence and predictors of hospitalization for bacterial infection in community-based patients with type 2 diabetes: the fremantle diabetes study. PLoS One. 2013;8(3):e60502. https ://doi.org/10.1371/journal.pone.0060502.

19. Peleg AY, Weerarathna T, McCarthy JS, Davis TM. Common infections in diabetes: pathogenesis, management and relationship 
to glycaemic control. Diabetes Metab Res Rev. 2007;23(1):3-13. https://doi.org/10.1002/dmrr.682.

20. McGuinness OP. Defective glucose homeostasis during infection. Annu Rev Nutr. 2005;25:9-35. https://doi.org/10.1146/annur ev.nutr.24.012003.132159.

21. Goldenberg RM, Berard LD, Cheng AY, Gilbert JD, Verma S, Woo VC, et al. SGLT2 inhibitor-associated diabetic ketoacidosis: clinical review and recommendations for prevention and diagnosis. Clin Ther. 2016;38(12):2654-2664 e1. https://doi. org/10.1016/j.clinthera.2016.11.002.

22. Wang Y, Desai M, Ryan PB, DeFalco FJ, Schuemie MJ, Stang PE, et al. Incidence of diabetic ketoacidosis among patients with type 2 diabetes mellitus treated with SGLT2 inhibitors and other antihyperglycemic agents. Diabetes Res Clin Pract. 2017;128:83-90. https://doi.org/10.1016/j.diabres.2017.04.004.
23. Neal B, Perkovic V, Matthews DR. Canagliflozin and cardiovascular and renal events in type 2 diabetes. N Engl J Med. 2017;377(21):2099. https://doi.org/10.1056/NEJMc1712572.

24. Wanner C, Inzucchi SE, Lachin JM, Fitchett D, von Eynatten M, Mattheus M, et al. Empagliflozin and progression of kidney disease in type 2 diabetes. N Engl J Med. 2016;375(4):323-34. https ://doi.org/10.1056/NEJMoa1515920.

25. Erondu N, Desai M, Ways K, Meininger G. Diabetic ketoacidosis and related events in the canagliflozin type 2 diabetes clinical program. Diabetes Care. 2015;38(9):1680-6. https://doi.org/10.2337/ dc15-1251. 